\title{
Cooperative AF Relaying in Spectrum-Sharing Systems: Outage Probability Analysis under Co-Channel Interferences and Relay Selection
}

\author{
Minghua Xia, Member, IEEE, and Sonia Aïssa, Senior Member, IEEE
}

\begin{abstract}
For cooperative amplify-and-forward (AF) relaying in spectrum-sharing wireless systems, secondary users share spectrum resources originally licensed to primary users to communicate with each other and, thus, the transmit power of secondary transmitters is strictly limited by the tolerable interference powers at primary receivers. Furthermore, the received signals at a relay and at a secondary receiver are inevitably interfered by the signals from primary transmitters. These co-channel interferences (CCIs) from concurrent primary transmission can significantly degrade the performance of secondary transmission. This paper studies the effect of CCIs on outage probability of the secondary link in a spectrum-sharing environment. In particular, in order to compensate the performance loss due to CCIs, the transmit powers of a secondary transmitter and its relaying node are respectively optimized with respect to both the tolerable interference powers at the primary receivers and the CCIs from the primary transmitters. Moreover, when multiple relays are available, the technique of opportunistic relay selection is exploited to further improve system performance with low implementation complexity. By analyzing lower and upper bounds on the outage probability of the secondary system, this study reveals that it is the tolerable interference powers at primary receivers that dominate the system performance, rather than the CCIs from primary transmitters. System designers will benefit from this result in planning and designing next-generation broadband spectrum-sharing systems.
\end{abstract}

Index Terms-Amplify-and-forward (AF) relaying, co-channel interference (CCI), interference power constraints, outage probability, relay selection, spectrum sharing.

\section{INTRODUCTION}

$\mathbf{S}$ SPECTRUM-SHARING cognitive radio is a promising wireless technique to address the increasing shortage of the indispensable electromagnetic spectrum resources [1]. In particular, as illustrated in Fig. 1-a), spectrum-sharing cognitive radio allows secondary users (SUs) to share spectrum resources originally licensed to primary users (PUs) as long as the interference power coming from SUs remains below a predefined tolerable threshold. Therefore, unlike other techniques with exclusive usage of spectrum resources, spectrum-sharing

Paper approved by Y. Chen, the Editor for Performance Analysis and Cognitive Radio of the IEEE Communications Society. Manuscript received February 7, 2012; revised April 17, 2012 and May 26, 2012.

This work was supported in part by King Abdullah University of Science and Technology (KAUST), Thuwal, Kingdom of Saudi Arabia (KSA).

M. Xia is with the Division of Physical Sciences and Engineering, KAUST, Thuwal, KSA (e-mail: minghua.xia@ieee.org).

S. Aïssa is with Institut National de la Recherche Scientifique (INRS), University of Quebec, Montreal, QC, Canada (e-mail: aissa@emt.inrs.ca).

Digital Object Identifier 10.1109/TCOMM.2012.09.xxxxx cognitive radio is more practical to be deployed in nextgeneration broadband wireless systems. Nevertheless, since the transmit power of SUs is dominated by the tolerable interference power at PUs, the coverage of secondary transmission in spectrum-sharing systems is strictly limited.

In order to extend the coverage of secondary transmission and/or enhance system performance in spectrum-sharing context, cooperative relaying techniques can be further exploited. As shown in Fig. 1-b), an intermediate relaying node between a transmitter and its destination can be used to assist the communication process. In general, there are two relaying protocols: decode-and-forward (DF) and amplify-andforward (AF). The performance of DF relaying in spectrumsharing context is widely investigated in the literature, see e.g. [2]-[5] and references therein. However, it is well-known that AF relaying has simpler structure than DF relaying and it can be more easily implemented in practice. In our recent work [6]-[8], system performance of cooperative AF relaying in spectrum-sharing environment is explored extensively. In particular, when multiple AF relays are available in spectrumsharing systems (cf. Fig. 1-c)), techniques of relay selection can be applied for higher spatial diversity gain [6], [8]. However, in practical systems, PUs and SUs can simultaneously transmit signals by sharing the same spectrum resources. As a result, the relays and secondary receiver inevitably suffer interferences from PUs. From the viewpoint of SUs, these interferences come in the form of co-channel interferences (CCIs) and their effect on system performance is important to be analyzed and quantified. This issue will be addressed in this paper.

Figure 1-d) illustrates the system model under study in this paper. Unlike the system in the absence of CCIs as shown in Fig. 1-c), there are two different categories of interference channels in Fig. 1-d). One pertains to the channels from the secondary transmitter or the relays to the primary receivers, which are indicated by red arrow lines in Fig. 1-d), i.e. $\mathrm{SU}_{\mathrm{t}} \rightarrow \mathrm{PU}_{\mathrm{r} 1}$ and $\mathrm{R}_{i} \rightarrow \mathrm{PU}_{\mathrm{r} 2}, i=1, \cdots, N$. These interference channels are related to the tolerable interference powers at primary receivers. The other category concerns the channels from primary transmitters to the relays or the secondary receiver, which are sketched by blue arrow lines in Fig. $1-$ d), i.e. $\mathrm{PU}_{\mathrm{t} 1} \rightarrow \mathrm{R}_{i}, i=1, \cdots, N$ and $\mathrm{PU}_{\mathrm{t} 2} \rightarrow \mathrm{SU}_{\mathrm{r}}$. These interference channels introduce CCIs to secondary link. Due to the presence of CCIs from concurrent primary transmission, the performance of secondary transmission in 
spectrum-sharing context will be degraded significantly, compared to that in Fig. 1-c). An explicit quantification of this degradation is critical for system designers. This is the main motivation for this paper.

In order to compensate the performance loss due to CCIs, techniques of optimal transmit power allocation at the secondary transmitter and the relays, and of relay selection are adopted in this paper. Specifically, when allocating transmit powers at either the secondary transmitter or the relays, the effects of desired channels, interference channels, tolerable interference powers at primary receivers, and CCIs from primary transmitters are all taken into account. On the other hand, since the transmit power of secondary transmitters is strictly limited in spectrum-sharing systems, techniques of relay selection can be exploited to obtain higher spatial diversity gain and improve system performance. In general, there are two relay-selection strategies: opportunistic relay selection [8] and partial relay selection [6]. The former chooses the relay that has the highest end-to-end (i.e. source $\rightarrow$ relay $\rightarrow$ destination) signal-tointerference-plus-noise ratio (SINR). The latter chooses the relay that achieves the highest SINR at either the source-relay hop or the relay-destination hop. Clearly, opportunistic relay selection outperforms partial relay selection since high SINR at one hop cannot guarantee high SINR at another hop.

The results of this study reveal that it is the tolerable interference powers at primary receivers that dominate the outage probability of the secondary system under consideration, rather than the CCIs from primary transmitters or the average signal-to-noise ratio (SNR) at the secondary transmitter. Furthermore, lower and upper bounds on the outage probability are developed in closed-form under single-relay and multirelay scenarios. Specifically, for the single-relay case, outage probability of the system is tight with the lower bound. On the other hand, for the multi-relay scenario, opportunistic relay selection can be exploited to improve outage probability significantly and it is highly predictable from the upper bound.

The rest of this paper is organized as follows. Section II details the system model. Section III discusses the criteria for optimal power allocation and relay selection. The distribution functions of the signal-to-interference ratios (SIRs) at two consecutive hops are derived in Section IV. The outage probability of the system with either single relay or multiple relays is analyzed in Section V and, finally, concluding remarks are provided in Section VI.

\section{SySTEM MODEL}

Figure 1-d) illustrates a spectrum-sharing cooperative relaying system in the presence of CCIs. In the system, the transmission between two pairs of PUs $\left(\mathrm{PU}_{\mathrm{t} 1} \rightarrow \mathrm{PU}_{\mathrm{r} 1}\right.$ and $\mathrm{PU}_{\mathrm{t} 2} \rightarrow \mathrm{PU}_{\mathrm{r} 2}$ ) occurs simultaneously with the transmission between a pair of SUs $\left(\mathrm{SU}_{\mathrm{t}} \rightarrow \mathrm{SU}_{\mathrm{r}}\right)$. Also, since the power of secondary transmitter $\mathrm{SU}_{\mathrm{t}}$ is strictly limited, $N$ intermediate SUs serve as AF relays $\left(\mathrm{R}_{i}, i=1, \cdots, N\right)$ to assist the communication process between $\mathrm{SU}_{\mathrm{t}}$ and its destination $\mathrm{SU}_{\mathrm{r}}$. It is assumed that all terminals in the system are equipped with single omnidirectional half-duplex antenna each.

In conventional cooperative relaying systems, either in the absence of CCI (cf. Fig. 1-b)) or in the presence of CCIs
[9], the power of a transmitter is generally limited by its own maximum output power. However, in spectrum-sharing cooperative relaying systems, SUs can communicate with each other only when the interference power at PUs remains below pre-defined tolerable levels. Hence, the transmit power of a SU and its relaying node must be dynamically optimized with respect to not only the desired channels (i.e. $\mathrm{SU}_{\mathrm{t}} \rightarrow \mathrm{R}_{i}$ and $\mathrm{R}_{i} \rightarrow \mathrm{SU}_{\mathrm{r}}, i=1, \cdots, N$, which are shown by the black arrow lines in Fig.1-d)) but also to the interference channels from the secondary transmitter and the relays to the primary receivers (i.e. $\mathrm{SU}_{\mathrm{t}} \rightarrow \mathrm{PU}_{\mathrm{r} 1}$ and $\mathrm{R}_{i} \rightarrow \mathrm{PU}_{\mathrm{r} 2}$, shown by the red arrow lines in Fig.1-d)). On the other hand, unlike the spectrum-sharing system in the absence of CCIs (cf. Fig. 1c)), the received signals of relay $\mathrm{R}_{i}$ in Fig. 1-d) are interfered by the transmit signals of primary user $\mathrm{PU}_{\mathrm{t} 1}$, which are shown by the blue arrow lines in Fig.1-d). Similarly, the received signals of $\mathrm{SU}_{\mathrm{r}}$ are interfered by the transmit signals of $\mathrm{PU}_{\mathrm{t} 2}$. In this work, all transmission channels are supposed to be independent of each other and to be subject to block Rayleigh fading. That is, the channels remain invariant during each data transmission block but vary from one block to another.

With the assistance of multiple intermediate AF relays, the data transmission between $\mathrm{SU}_{\mathrm{t}}$ and $\mathrm{SU}_{\mathrm{r}}$ is performed in two consecutive phases. During the first-hop phase, secondary transmitter $\mathrm{SU}_{\mathrm{t}}$ sends signal $x$ with optimal transmit power $P_{1}$ (to be determined in the next section) to all relays and, meanwhile, primary transmitter $\mathrm{PU}_{\mathrm{t} 1}$ sends signal $s_{1}$ with fixed power $P_{s 1}$ to primary receiver $\mathrm{PU}_{\mathrm{r} 1} \cdot{ }^{1}$ Consequently, when the CCI from $\mathrm{PU}_{\mathrm{t} 1}$ is considered, the received signal at the $i^{\text {th }}$ relay $\left(\mathrm{R}_{i}, 1 \leq i \leq N\right)$ is given by

$$
y_{i}=\sqrt{P_{1}} f_{i} x+\sqrt{P_{s 1}} q_{i} s_{1}+n_{i},
$$

where $f_{i}$ and $q_{i}$ denote the coefficients of the desired channel from $\mathrm{SU}_{\mathrm{t}}$ to $\mathrm{R}_{i}$ and of the interference channel from $\mathrm{PU}_{\mathrm{t} 1}$ to $\mathrm{R}_{i}$ respectively, and $n_{i}$ is the additive white Gaussian noise (AWGN) at relay $\mathrm{R}_{i}$ with zero mean and variance $\sigma_{i}^{2}$. For ease of notation and without loss of generality, it is assumed throughout the rest of the paper that the AWGNs at all relays and at the secondary receiver have the same variance $\sigma^{2}$.

During the second-hop phase, the relay $\mathrm{R}_{i}$ amplifies its received signal with a power gain $\beta_{i}$ and forwards it to the secondary receiver with optimal transmit power $P_{2}$ (to be determined in the next section). Meanwhile, primary transmitter $\mathrm{PU}_{\mathrm{t} 2}$ sends signal $s_{2}$ with fixed power $P_{s 2}$ to primary receiver $\mathrm{PU}_{\mathrm{r} 2}$. As a consequence, when the $\mathrm{CCI}$ from $\mathrm{PU}_{\mathrm{t} 2}$ is taken into account, the received signal at secondary receiver $\mathrm{SU}_{\mathrm{r}}$ is given by

$$
\begin{aligned}
y= & \sqrt{P_{2}} g_{i} \beta_{i}\left(\sqrt{P_{1}} f_{i} x+\sqrt{P_{s 1}} q_{i} s_{1}+n_{i}\right) \\
& +\sqrt{P_{s 2}} q_{0} s_{2}+n,
\end{aligned}
$$

where $g_{i}$ and $q_{0}$ designate the coefficients of the desired channel from $\mathrm{R}_{i}$ to $\mathrm{SU}_{\mathrm{r}}$ and of the interference channel from

\footnotetext{
${ }^{1}$ Since this paper aims at evaluating the performance of cooperative relaying transmission in spectrum-sharing context, the power of the secondary transmitter will be optimized whereas the powers of the primary transmitters are assumed to be fixed. Primary transmitters introduce CCIs at the relays and at the secondary receiver and their effects on the performance of the secondary link will be studied by setting different power values in the simulation experiments presented in Section V.
} 


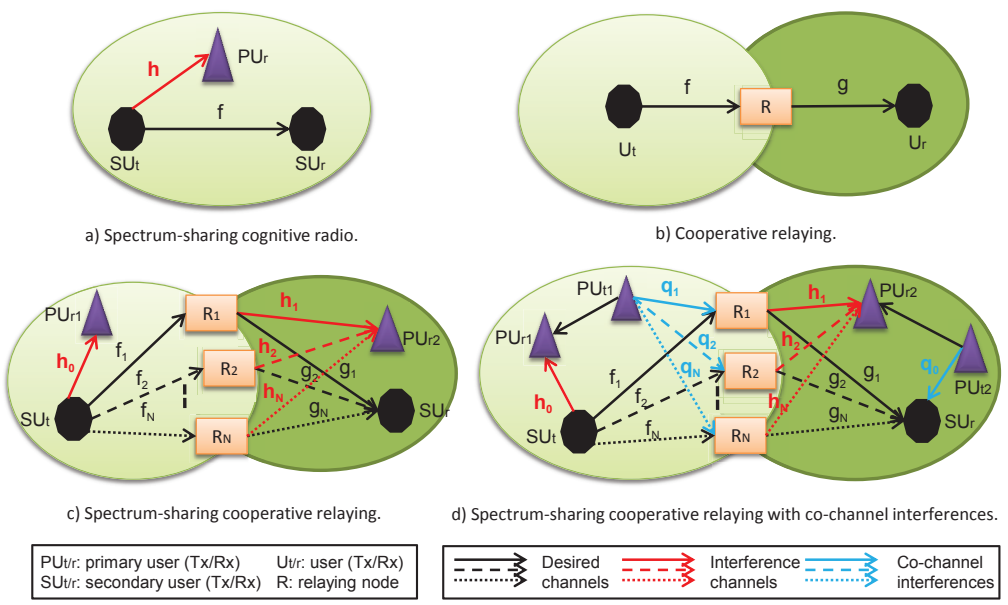

Fig. 1. The evolution of spectrum-sharing cooperative relaying systems.

$\mathrm{PU}_{\mathrm{t} 2}$ to $\mathrm{SU}_{\mathrm{r}}$ respectively, and $n$ stands for the AWGN at $\mathrm{SU}_{\mathrm{r}}$ with zero mean and variance $\sigma^{2}$. Also, without loss of generality, it is assumed that the amplitudes of all transmit signals (i.e. $x, s_{1}$, and $s_{2}$ ) are identical. In the sequel, two different choices of the power gain $\beta_{i}$ are made explicitly.

For AF relaying technique, the relay gain aims to invert the first-hop channel while limiting the output power of the relay when the channel-fading amplitude at the first hop is low. In general, there are two methods to determine the relay gain:

1) When full channel state information (CSI) is available at relay $R_{i}$, that is, when CSI of both the desired channel $\mathrm{SU}_{\mathrm{t}} \rightarrow \mathrm{R}_{i}$ and the interference channel $\mathrm{PU}_{\mathrm{t} 1} \rightarrow \mathrm{R}_{i}$ are known at relay $R_{i}$, the relay gain is determined by

$$
\beta_{i}=\left(P_{1}\left|f_{i}\right|^{2}+P_{s 1}\left|q_{i}\right|^{2}+\sigma^{2}\right)^{-\frac{1}{2}}
$$

where the operator $|x|$ stands for the amplitude of $x$.

2) When only partial CSI is available at relay $R_{i}$, that is, when only the CSI of the desired channel $\mathrm{SU}_{\mathrm{t}} \rightarrow \mathrm{R}_{i}$ is known at relay $R_{i}$, the relay gain is determined by

$$
\beta_{i}=\left(\sqrt{P_{1}} f_{i}\right)^{-1} \text {. }
$$

Substituting (3) into (2) and performing some straightforward algebraic manipulations, we obtain the end-to-end $\left(\mathrm{SU}_{\mathrm{t}} \rightarrow \mathrm{R}_{i} \rightarrow \mathrm{SU}_{\mathrm{r}}\right) \mathrm{SINR}$, and it is shown to be given by

$$
\gamma_{i}^{\prime}=\frac{\gamma_{\mathrm{a} i} \gamma_{\mathrm{b} i}}{\gamma_{\mathrm{a} i}+\gamma_{\mathrm{b} i}+1},
$$

where $\gamma_{\mathrm{a} i}$ and $\gamma_{\mathrm{b} i}$ stand for the received SINRs at the first hop $\left(\mathrm{SU}_{\mathrm{t}} \rightarrow \mathrm{R}_{i}\right)$ and at the second hop $\left(\mathrm{R}_{i} \rightarrow \mathrm{SU}_{\mathrm{r}}\right)$ respectively, defined by

$$
\gamma_{\mathrm{a} i} \triangleq \frac{P_{1}\left|f_{i}\right|^{2}}{P_{s 1}\left|q_{i}\right|^{2}+\sigma^{2}}, \quad \gamma_{\mathrm{b} i} \triangleq \frac{P_{2}\left|g_{i}\right|^{2}}{P_{s 2}\left|q_{0}\right|^{2}+\sigma^{2}} .
$$

Similarly, substituting (4) into (2) and performing some algebraic manipulations, we obtain the end-to-end signal-tointerference ratio (SIR) and it is shown to be given by

$$
\gamma_{i}=\frac{\gamma_{1 i} \gamma_{2 i}}{\gamma_{1 i}+\gamma_{2 i}},
$$

where $\gamma_{1 i}$ and $\gamma_{2 i}$ represent the received SIRs at the first hop and at the second hop respectively, defined by

$$
\gamma_{1 i} \triangleq \frac{P_{1}\left|f_{i}\right|^{2}}{P_{s 1}\left|q_{i}\right|^{2}}, \quad \gamma_{2 i} \triangleq \frac{P_{2}\left|g_{i}\right|^{2}}{P_{s 2}\left|q_{0}\right|^{2}} .
$$

When the CCIs from PUs are considered, system performance of secondary transmission in spectrum-sharing context is dominated by the interference rather than noise. In essence, the received SIR given by (7) is an upper bound on the SINR in (5) and it becomes tight when the interference-to-noise ratio is medium or high. Moreover, (7) has the advantage of mathematical tractability over (5). Therefore, hereafter the system performance analysis is based on the SIR expression in (7).

\section{CRiteria for Power Allocation AND Relay SELECTION}

In this section, the criteria for optimal transmit power allocation (at either secondary source $\mathrm{SU}_{\mathrm{t}}$ or relaying node $\mathrm{R}_{i}$ ) and for relay selection are established.

\section{A. Criteria for Optimal Power Allocation}

In this subsection, the criteria for optimal transmit powers ( $P_{1}$ at secondary transmitter $\mathrm{SU}_{\mathrm{t}}$ and $P_{2}$ at relaying node $\mathrm{R}_{i}$ ) are developed. Provided that relay $\mathrm{R}_{i}$ is chosen out of the $N$ available relays (the relay-selection criterion will be discussed in the next subsection), in order to achieve the channel capacity $C_{1}$ at the first hop $\left(\mathrm{SU}_{\mathrm{t}} \rightarrow \mathrm{R}_{i}\right)$, by recalling the received SIR $\gamma_{1 i}$ in (8), the optimal transmit power $P_{1}$ at $\mathrm{SU}_{\mathrm{t}}$ is determined as the solution to the optimization problem:

$$
\begin{gathered}
C_{1}=\max _{P_{1} \geq 0} \mathcal{E}_{P_{1}, f_{i}, q_{i}}\left\{\log _{2}\left(1+\frac{P_{1}\left|f_{i}\right|^{2}}{P_{s 1}\left|q_{i}\right|^{2}}\right)\right\} \\
\text { s.t. } \quad \mathcal{E}_{P_{1}, h_{0}}\left\{P_{1}\left|h_{0}\right|^{2}\right\} \leq W .
\end{gathered}
$$

where the operator $\mathcal{E}_{X}\{$.$\} denotes mathematical expectation$ associated with $X, h_{0}$ is the coefficient of interference channel from the secondary transmitter to primary receiver $\mathrm{PU}_{\mathrm{r} 1}$, and $W$ is the average tolerable interference power at $\mathrm{PU}_{\mathrm{r} 1}$. Clearly, the optimal transmit power $P_{1}$ of the secondary transmitter is a function of $f_{i}, q_{i}, h_{0}, P_{s 1}$, and $W$, which will be explicitly determined below. 
In general, the tolerable interference power at PUs can be defined by means of average interference power, peak interference power, or both [10], [11]. The average interference power constraint has low feedback overhead and it applies to non-real time applications where the quality-ofservice (QoS) depends upon the average output SNR. The peak interference power constraint has high feedback overhead since it requires instantaneous CSI of interference channels to determine the transmit power of SUs, and it is appropriate for real-time applications. Moreover, the transmit power of a SU is physically limited by its own maximum output power, which is essentially equivalent to the peak interference power constraint. Nevertheless, it was previously demonstrated that peak interference-power constraint will not affect system performance when it is large enough [11], [12]. Therefore, we consider only the average interference power constraint in the optimization problem above.

Applying the Lagrangian optimization technique to (9a)(9b), it is straightforward to show that the optimal transmit power of the secondary transmitter is given by

$$
P_{1}=\left[\frac{\lambda_{1}}{\left|h_{0}\right|^{2}}-P_{s 1} \frac{\left|q_{i}\right|^{2}}{\left|f_{i}\right|^{2}}\right]^{+},
$$

where the operator $[x]^{+} \triangleq \max (0, x)$ and the parameter $\lambda_{1}$ is determined by the average interference power constraint satisfying the equality in $(9 b)$, such that

$$
\mathcal{E}_{h_{0}, f_{i}, q_{i}}\left\{\left[\lambda_{1}-P_{s 1} \frac{\left|h_{0}\right|^{2}\left|q_{i}\right|^{2}}{\left|f_{i}\right|^{2}}\right]^{+}\right\}=W .
$$

It is worth pointing out that the optimal power allocation in (9c) performs like the well-known water-filling power allocation algorithm constrained by the maximum transmit power [13]. The parameter $\lambda_{1}$ is known as water-level and it will be explicitly determined in Section IV-A below. The operator $[x]^{+}$implies that the transmit power $P_{1}$ is zero if the strength of the desired channel $\left|f_{i}\right|^{2} \leq \frac{P_{s 1}}{\lambda_{1}}\left|h_{0}\right|^{2}\left|q_{i}\right|^{2}$, namely, no data will be transmitted in such case. In other words, only when $\left|f_{i}\right|^{2}>\frac{P_{s 1}}{\lambda_{1}}\left|h_{0}\right|^{2}\left|q_{i}\right|^{2}$ can $\mathrm{SU}_{\mathrm{t}}$ transmit to all relays, which makes the cooperative relaying transmission in spectrum-sharing context completely different from conventional cooperative relaying systems where the transmitter can send data to its relay at any time, regardless of the channel conditions between them.

During the second-hop phase, the relay $\mathrm{R}_{i}$ that is chosen out of the $N$ available relays forwards the received signal with optimal transmit power $P_{2}$, such that it achieves the channel capacity at the second hop while constrained by the average tolerable interference power $W$ at primary receiver $\mathrm{PU}_{\mathrm{r} 2}$. It is worthwhile mentioning that, in this paper, the average tolerable interference powers at primary receivers $\mathrm{PU}_{\mathrm{r} 1}$ and $\mathrm{PU}_{\mathrm{r} 2}$ are assumed to be symmetric (i.e. $W_{1}=W_{2}=W$ ), because this system outperforms that with non-symmetric interference power constraint (i.e. $W_{1} \neq W_{2}$ ) provided that the sum of $W_{1}$ and $W_{2}$ is fixed [8]. Therefore, according to the received SIR $\gamma_{2 i}$ in (8), $P_{2}$ satisfies the optimization problem:

$$
C_{2}=\max _{P_{2} \geq 0} \mathcal{E}_{P_{2}, g_{i}, q_{0}}\left\{\log _{2}\left(1+\frac{P_{2}\left|g_{i}\right|^{2}}{P_{s 2}\left|q_{0}\right|^{2}}\right)\right\}
$$

$$
\text { s.t. } \mathcal{E}_{P_{2}, h_{i}}\left\{P_{2}\left|h_{i}\right|^{2}\right\} \leq W
$$

where $h_{i}$ denotes the complex coefficient of the interferencechannel from relay $\mathrm{R}_{i}$ to primary receiver $\mathrm{PU}_{\mathrm{r} 2}$. Using a similar approach as in the fist-hop phase, $P_{2}$ is found to be given by

$$
P_{2}=\left[\frac{\lambda_{2}}{\left|h_{i}\right|^{2}}-P_{s 2} \frac{\left|q_{0}\right|^{2}}{\left|g_{i}\right|^{2}}\right]^{+},
$$

where the parameter $\lambda_{2}$ is determined by

$$
\mathcal{E}_{q_{0}, h_{i}, g_{i}}\left\{\left[\lambda_{2}-P_{s 2} \frac{\left|q_{0}\right|^{2}\left|h_{i}\right|^{2}}{\left|g_{i}\right|^{2}}\right]^{+}\right\}=W .
$$

It is noteworthy that the instantaneous CSI of interference channels, namely, $h_{0}$ and $q_{i}$ in (9c) and $q_{0}$ and $h_{i}$ in (10c), can be obtained at the SUs by periodic sounding of the pilot signals transmitted by the PUs. Although the acquisition of these CSI requires additional cost at the SUs, it enables them to strictly abide by the interference power constraints dictated by the PUs and to optimize system performance of the secondary transmission.

\section{B. Criteria for Relay Selection}

When multiple relays are available to cooperate between secondary transmitter $\mathrm{SU}_{\mathrm{t}}$ and its receiver $\mathrm{SU}_{\mathrm{r}}$, multi-relay transmission in parallel incurs severe interference at PUs and they have to work on orthogonal channels in order to avoid interference with each other. Therefore, techniques of relay selection can be further exploited in practical systems to leverage spatial diversity gain while alleviating interference at PUs. That is, only the "best" relay is chosen to assist $\mathrm{SU}_{\mathrm{t}}$ in transmitting to $\mathrm{SU}_{\mathrm{r}}$ while the other relays stay silent.

In general, there are mainly two relay-selection strategies [8], [14], [15]. One is opportunistic relay selection, which is based on the end-to-end SIRs. The other is partial relay selection, which is based on single-hop SIRs (either the first hop or the second hop). It was previously demonstrated that opportunistic relay selection achieves the diversity gain $G_{d}=$ $N$ whereas partial relay selection has diversity gain $G_{d}=1$ [8]. Therefore, in this paper, opportunistic relay selection is adopted to attain better system performance. Mathematically, the index $\hat{i}$ of the chosen relay is determined by

$$
\hat{i}=\arg \max _{i=1, \cdots, N}\left\{\gamma_{i}\right\},
$$

where $\gamma_{i}$ is given by (7)

\section{Distributions Functions of The SIRs AT Consecutive Hops}

In this section, we first determine the optimal transmit powers at the secondary transmitter and the relays. Once the power allocation completed, the distribution functions of the SIRs at two consecutive hops are developed in closed-form.

\section{A. Optimal Transmit Powers $P_{1}$ and $P_{2}$}

Based on the power-allocation criteria described in Section III-A, in order to determine the optimal transmit powers $P_{1}$ and $P_{2}$, first we need to determine the optimal power allocation parameters $\lambda_{1}$ and $\lambda_{2}$. By virtue of (9d), in order 
to determine the value of $\lambda_{1}$ we derive the probability density function (PDF) of $V \triangleq \frac{\left|h_{0}\right|^{2}\left|q_{i}\right|^{2}}{\left|f_{i}\right|^{2}}$. Since all channels are supposed to be subject to Rayleigh fading with the same mean $\bar{\gamma}$, the PDFs of $\left|h_{0}\right|^{2},\left|q_{i}\right|^{2}$, and $\left|f_{i}\right|^{2}$ are in the form of

$$
f_{X}(x)=\frac{1}{\bar{\gamma}} \exp \left(-\frac{x}{\bar{\gamma}}\right), \quad X \in\left\{\left|h_{0}\right|^{2},\left|q_{i}\right|^{2},\left|f_{i}\right|^{2}\right\} \text {. }
$$

After some tedious but straightforward algebraic derivations, the PDF of $V$ is obtained as in the following lemma.

Lemma 1: If three independent channel gains $\left|h_{0}\right|^{2},\left|q_{i}\right|^{2}$, and $\left|f_{i}\right|^{2}$ are of Rayleigh distribution with the same mean $\bar{\gamma}$, then the PDF of $V=\frac{\left|h_{0}\right|^{2}\left|q_{i}\right|^{2}}{\left|f_{i}\right|^{2}}$ is given by

$$
f_{V}(x)=\frac{1}{\bar{\gamma}} \Psi\left(2,1 ; \frac{x}{\bar{\gamma}}\right),
$$

where $\Psi(a, b ; x)$ denotes the Tricomi confluent hypergeometric function [16, Eq.(9.210.2)].

Proof: See Appendix A.

Before proceeding further, we introduce the following integral equality associated with the Meijer's $G$-function, which will be applied repeatedly in the sequel.

Lemma 2: For $c, \alpha>0$, the following integral equality holds

$$
\int_{0}^{c} x^{\alpha-1} G_{p, q}^{m, n}\left[x \mid \begin{array}{c}
\left(a_{p}\right) \\
\left(b_{q}\right)
\end{array}\right] \mathrm{d} x=G_{p+1, q+1}^{m, n+1}\left[c \mid \begin{array}{c}
1,\left(a_{p}+\alpha\right) \\
\left(b_{q}+\alpha\right), 0
\end{array}\right],
$$

where $G[x \mid$.$] denotes the Meijer's G$-function $[16$, Eq.(9.301)].

Proof: By virtue of [17, vol.3, Eq.(2.24.2.2)] and the scaling property of Meijer's $G$-function [17, vol.3, Eq.(8.2.2.15)], (14) can be derived in a straightforward manner.

After obtaining the PDF of $V$ in (13) and substituting it into (9d), the power allocation parameter $\lambda_{1}$ can be shown to be determined by (15) at the top of the next page, where $c_{1} \triangleq \frac{\lambda_{1}}{\bar{\gamma} P_{s 1}}$. Subsequently, by expressing Tricomi confluent hypergeometric function in the integrand of (15) in terms of Meijer's $G$-function [17, vol.3, Eq.(8.4.46.1)]:

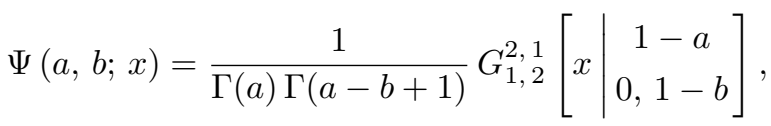

(15) can be rewritten as (17)-(19) at the top of the next page, where (14) was used to derive (18), and the definition of $c_{1}$ immediately after (15) was substituted into (18) and the scaling property of Meijer's $G$-function [17, vol.3, Eq.(8.2.2.15)] was exploited to reach (19). It is noteworthy that (19) implies that, when the average tolerable interference power $W$ at PUs is fixed, the value of the power-allocation parameter $\lambda_{1}$ depends only upon the product of $\bar{\gamma}$ and $P_{s 1}$. That is, increasing the mean $\bar{\gamma}$ of desired-channel gain is equivalent to decreasing the power $P_{s 1}$ of CCI, and vice versa. This provides high flexibility to improve system performance of secondary transmission in spectrum-sharing context.

Although the power-allocation parameter $\lambda_{1}$ in (19) cannot be explicitly expressed in closed-form, it can be easily obtained in a numerical way because Meijer's $G$-function is a built-in function in popular mathematical softwares, such as Matlab and Mathematica. Moreover, since the tolerable interference powers at the primary receivers are symmetric (i.e. $W_{1}=W_{2}=W$ ), it is straightforward that the power allocation parameter at the second hop is identical to that at the first hop, that is, $\lambda_{2}=\lambda_{1}$. With the values of $\lambda_{1}$ and $\lambda_{2}$ established, the optimal transmit powers $P_{1}$ and $P_{2}$ can be readily determined by using (9c) and (10c), respectively. Afterwards, with the resultant $P_{1}$ and $P_{2}$, we derive the distribution functions of the SIRs at consecutive hops in the next subsection.

\section{B. Distribution Functions of the SIRs $\gamma_{1 i}$ and $\gamma_{2 i}$}

Substituting $P_{1}$ in (9c) into the expression of $\gamma_{1 i}$ in (8), the SIR pertaining to the first hop can be given by

$$
\gamma_{1 i}=\left[\frac{\lambda_{1}}{P_{s 1}} \frac{\left|f_{i}\right|^{2}}{\left|h_{0}\right|^{2}\left|q_{i}\right|^{2}}-1\right]^{+}=\left[\frac{\lambda_{1}}{P_{s 1}} V^{\prime}-1\right]^{+},
$$

where $V^{\prime} \triangleq \frac{\left|f_{i}\right|^{2}}{\left|h_{0}\right|^{2}\left|q_{i}\right|^{2}}=\frac{1}{V}$. Upon using a similar approach as in the proof of Lemma 1, the PDF of $V^{\prime}$ can be shown to be expressed as

$$
f_{V^{\prime}}(x)=\frac{1}{\bar{\gamma} x^{2}} \Psi\left(2,1 ; \frac{1}{\bar{\gamma} x}\right) .
$$

In view of (20)-(21) and performing some tedious mathematical derivations by means of the powerful Meijer's $G$ function, the distribution functions of $\gamma_{1 i}$ can be obtained in compact forms and they are given in the following theorem.

Theorem 1: For the spectrum-sharing cooperative AF relaying system in the presence of CCIs, the PDF and CDF of the received SIR at the first hop, i.e. $\gamma_{1 i}$ in (20), are given by

$$
f_{\gamma_{1 i}}(\gamma)=\frac{1}{(\gamma+1)^{2} G_{1}} \Psi\left(2,1 ; \frac{c_{1}}{\gamma+1}\right)
$$

and

$$
F_{\gamma_{1 i}}(\gamma)=1-\frac{1}{c_{1} G_{1}} G_{2,3}^{2,2}\left[\begin{array}{c|c}
\frac{c_{1}}{\gamma+1} & 1,0 \\
1,1,0
\end{array}\right],
$$

respectively, where the parameter $G_{1}$ is determined by

$$
G_{1} \triangleq G_{2,3}^{2,2}\left[\begin{array}{c|c}
0,-1 \\
c_{1} \\
0,0,-1
\end{array}\right] .
$$

Proof: See Appendix B.

Using a similar approach as in the proof of Theorem 1, the distribution functions of $\gamma_{2 i}$ can be derived and are summarized in the following theorem.

Theorem 2: For the spectrum-sharing cooperative AF relaying system in the presence of CCIs, the PDF and CDF of the received SIR at the second hop, i.e. $\gamma_{2 i}$ in (8), are given by

$$
f_{\gamma_{2 i}}(\gamma)=\frac{1}{(\gamma+1)^{2} G_{2}} \Psi\left(2,1 ; \frac{c_{2}}{\gamma+1}\right)
$$

and

$$
F_{\gamma_{2 i}}(\gamma)=1-\frac{1}{c_{2} G_{2}} G_{2,3}^{2,2}\left[\begin{array}{c|c}
c_{2} & 1,0 \\
\gamma+1 & 1,1,0
\end{array}\right],
$$

respectively, where the parameters $c_{2}$ and $G_{2}$ are defined as

$$
c_{2} \triangleq \frac{\lambda_{2}}{\bar{\gamma} P_{s 2}}
$$




$$
\begin{aligned}
W & =\int_{0}^{\frac{\lambda_{1}}{P_{s 1}}}\left(\lambda_{1}-x P_{s 1}\right) f_{V}(x) \mathrm{d} x \\
& =\frac{\lambda_{1}}{\bar{\gamma}} \int_{0}^{\frac{\lambda_{1}}{P_{s 1}}} \Psi\left(2,1 ; \frac{x}{\bar{\gamma}}\right) \mathrm{d} x-\frac{P_{s 1}}{\bar{\gamma}} \int_{0}^{\frac{\lambda_{1}}{P_{s 1}}} x \Psi\left(2,1 ; \frac{x}{\bar{\gamma}}\right) \mathrm{d} x \\
& =\lambda_{1} \int_{0}^{c_{1}} \Psi(2,1 ; x) \mathrm{d} x-\bar{\gamma} P_{s 1} \int_{0}^{c_{1}} x \Psi(2,1 ; x) \mathrm{d} x,
\end{aligned}
$$

$$
\begin{aligned}
& W=\lambda_{1} \int_{0}^{c_{1}} G_{1,2}^{2,1}\left[x \mid \begin{array}{c}
-1 \\
0,0
\end{array}\right] \mathrm{d} x-\bar{\gamma} P_{s 1} \int_{0}^{c_{1}} x G_{1,2}^{2,1}\left[x \mid \begin{array}{c}
-1 \\
0,0
\end{array}\right] \mathrm{d} x \\
& =\lambda_{1} G_{2,3}^{2,2}\left[c_{1} \mid \begin{array}{c}
1,0 \\
1,1,0
\end{array}\right]-\bar{\gamma} P_{s 1} G_{2,3}^{2,2}\left[\begin{array}{c|c}
c_{1} & \left.\begin{array}{c}
1,1 \\
2,2,0
\end{array}\right]
\end{array}\right. \\
& =\bar{\gamma} P_{s 1}\left\{G_{2,3}^{2,2}\left[\begin{array}{c|c}
\frac{\lambda_{1}}{\bar{\gamma} P_{s 1}} & 2,1 \\
2,2,1
\end{array}\right]-G_{2,3}^{2,2}\left[\begin{array}{c|c}
\lambda_{1} & 1,1 \\
\bar{\gamma} P_{s 1} & 2,2,0
\end{array}\right]\right\} \text {, }
\end{aligned}
$$

$$
G_{2} \triangleq G_{2,3}^{2,2}\left[\begin{array}{l|c}
0,-1 \\
c_{2} \\
0,0,-1
\end{array}\right] .
$$

With the distribution functions of $\gamma_{1 i}$ and $\gamma_{2 i}$ established, in the next section we analyze the outage probability of the end-to-end SIR at the secondary receiver.

\section{Analysis of Outage Probability}

Due to the high complexity of the distribution functions of the SIRs at consecutive hops as stated in Theorems 1 and 2 above, it is mathematically intractable to derive the exact distribution functions of the end-to-end SIR given in (7). Therefore, in this section we analyze the lower and upper bounds on the outage probability for single-relay and multirelay scenarios, so as to gain insight into system performance.

\section{A. Single-Relay Scenario}

We start by looking at the lower and upper bounds of the end-to-end SIR $\gamma_{i}$. In view of (7), we recognize that [18]

$$
\underbrace{\frac{1}{2} \min \left\{\gamma_{1 i}, \gamma_{2 i}\right\}}_{\gamma_{i-\text { low }}} \leq \gamma_{i}=\frac{\gamma_{1 i} \gamma_{2 i}}{\gamma_{1 i}+\gamma_{2 i}} \leq \underbrace{\min \left\{\gamma_{1 i}, \gamma_{2 i}\right\}}_{\gamma_{i-\text { up }}},
$$

where the lower bound is achieved when $\gamma_{1 i}$ and $\gamma_{2 i}$ are symmetric (i.e. $\gamma_{1 i}=\gamma_{2 i}$ ) and the upper bound is achieved when $\gamma_{1 i}$ and $\gamma_{2 i}$ are non-symmetric (i.e. $\gamma_{1 i} \gg \gamma_{2 i}$ or $\left.\gamma_{1 i} \ll \gamma_{2 i}\right)$. Notice that, for notational consistency, we still use $\gamma_{i}$ to express the end-to-end SIR even when there is only a single relay.

The outage probability is defined as the probability that the instantaneous end-to-end SIR falls below a predefined threshold value $\gamma_{\text {th }}$. Mathematically, the outage probability is expressed as

$$
\mathrm{P}_{\text {outage }}^{\text {single }}\left(\gamma_{\mathrm{th}}\right)=\operatorname{Pr}\left\{\gamma_{i}<\gamma_{\mathrm{th}}\right\}=F_{\gamma_{i}}\left(\gamma_{\mathrm{th}}\right),
$$

where the superscript "single" of $\mathrm{P}_{\text {outage }}^{\text {single }}\left(\gamma_{\mathrm{th}}\right)$ stands for the single-relay case. Intuitively, (30) means that the outage probability can be obtained by evaluating the CDF of the end-to-end SIR at the threshold $\gamma_{\mathrm{th}}$. Moreover, since outage probability is a monotonically decreasing function of the endto-end SIR, substituting (29) into (30) yields the lower and upper bounds on outage probability. That is,

$$
F_{\gamma_{i-\text { up }}}\left(\gamma_{\text {th }}\right) \leq \mathrm{P}_{\text {outage }}^{\text {single }}\left(\gamma_{\text {th }}\right) \leq F_{\gamma_{i-\text { low }}}\left(\gamma_{\text {th }}\right)
$$

Since the SIRs $\gamma_{1 i}$ and $\gamma_{2 i}$ at consecutive hops are independent of each other, by recalling the theory of order statistics, it is straightforward to show that the CDF of $\gamma_{i-\text { up }} \triangleq \min \left\{\gamma_{1 i}, \gamma_{2 i}\right\}$ is given by

$$
\begin{aligned}
F_{\gamma_{i-\text { up }}}(\gamma) & =1-\left[1-F_{\gamma_{1 i}}(\gamma)\right]\left[1-F_{\gamma_{2 i}}(\gamma)\right] \\
& =F_{\gamma_{1 i}}(\gamma)+F_{\gamma_{2 i}}(\gamma)-F_{\gamma_{1 i}}(\gamma) F_{\gamma_{2 i}}(\gamma) .(32)
\end{aligned}
$$

On the other hand, in light of (29), it is evident that $\gamma_{i-\text { low }}=$ $\frac{1}{2} \gamma_{i-\text { up }}$. Hence, by virtue of (32), the CDF of $\gamma_{i-\text { low }}$ can be expressed as

$$
F_{\gamma_{i-\mathrm{low}}}(\gamma)=F_{\gamma_{1 i}}(2 \gamma)+F_{\gamma_{2 i}}(2 \gamma)-F_{\gamma_{1 i}}(2 \gamma) F_{\gamma_{2 i}}(2 \gamma)
$$

Consequently, substituting (32) and (33) into (30) gives us the lower and upper bounds on outage probability and they are

$$
\begin{gathered}
F_{\gamma_{1 i}}\left(\gamma_{\mathrm{th}}\right)+F_{\gamma_{2 i}}\left(\gamma_{\mathrm{th}}\right)-F_{\gamma_{1 i}}\left(\gamma_{\mathrm{th}}\right) F_{\gamma_{2 i}}\left(\gamma_{\mathrm{th}}\right) \\
\leq \mathrm{P}_{\text {outage }}^{\text {single }}\left(\gamma_{\mathrm{th}}\right) \leq \\
\quad F_{\gamma_{1 i}}\left(2 \gamma_{\mathrm{th}}\right)+F_{\gamma_{2 i}}\left(2 \gamma_{\mathrm{th}}\right)-F_{\gamma_{1 i}}\left(2 \gamma_{\mathrm{th}}\right) F_{\gamma_{2 i}}\left(2 \gamma_{\mathrm{th}}\right) .
\end{gathered}
$$

It will be shown below that simulation results of outage probability is very tight with the lower bound computed by (34). Furthermore, in order to gain insights into system performance, we investigate the effects of tolerable interference power, average SNR and CCI on the outage probability by deriving the diversity order and the coding gain as follows. Specifically, by virtue of (34), the CDF of the received SIR can be approximately given by the lower bound and it is dominated by the worse link between consecutive hops, that is, $F_{\gamma_{i}}(\gamma) \approx \max \left\{F_{\gamma_{1 i}}(\gamma), F_{\gamma_{2 i}}(\gamma)\right\}$. Without loss of generality, assuming $F_{\gamma_{1 i}}(\gamma)>F_{\gamma_{2 i}}(\gamma)$ leads to $F_{\gamma_{i}}(\gamma) \approx F_{\gamma_{1 i}}(\gamma)$. Thus, 
the PDF of the received SIR can be approximately given by $f_{\gamma_{i}}(\gamma) \approx f_{\gamma_{1 i}}(\gamma)$. Then, according to [20], if the limit of the PDF of the received SIR can be expressed as:

$$
\lim _{\gamma \rightarrow 0} f_{\gamma_{i}}(\gamma)=a \gamma^{t}+o\left(\gamma^{t+\epsilon}\right)
$$

where $a, \epsilon>0$ and $o($.$) pertain to the Landau notation, then$ the diversity order $G_{d}$ is given by

$$
G_{d}=t+1
$$

and the coding gain is proportional to $a^{-\frac{1}{t+1}}$, i.e.

$$
G_{c} \sim a^{-\frac{1}{t+1}} .
$$

In light of (22), it is straightforward that

$$
\begin{aligned}
\lim _{\gamma \rightarrow 0} f_{\gamma_{1 i}}(\gamma) & =\frac{1}{G_{1}} \Psi\left(2,1 ; c_{1}\right) \\
& =\frac{-1+\left(1+c_{1}\right) e^{c_{1}} \Gamma\left(0, c_{1}\right)}{e^{c_{1}} \Gamma\left(0, c_{1}\right)} \\
& =1+c_{1}-\frac{e^{-c_{1}}}{\Gamma\left(0, c_{1}\right)} .
\end{aligned}
$$

At high SNR (i.e. large $\bar{\gamma}$ ), $c_{1}=\frac{\lambda_{1}}{\bar{\gamma} P_{s 1}} \rightarrow 0$, and we have $\Gamma\left(0, c_{1}\right) \sim-\ln c_{1}$ [21, Eq. (3.42)] and $e^{-c_{1}} \sim 1-c_{1}$. Substituting them into (38) leads to

$$
\begin{aligned}
\lim _{\gamma \rightarrow 0} f_{\gamma_{1 i}}(\gamma) & =1+c_{1}+\frac{1-c_{1}}{\ln c_{1}} \\
& \sim 1-c_{1} .
\end{aligned}
$$

Consequently, applying (35)-(37) to (39) yields

$$
G_{d}=1
$$

and

$$
G_{c} \sim 1+\frac{\lambda_{1}}{\bar{\gamma} P_{s 1}},
$$

where the value of $c_{1}$ shown immediately after (15) was substituted into (39) to reach (41). Clearly, (41) shows that the coding gain increases with $\lambda_{1}$ while decreases with $\bar{\gamma}$ and $P_{s 1}$. This implies that the power-allocation parameter $\lambda_{1}$ has more significant effect on coding gain than the average SNR $(\bar{\gamma})$ and CCI $\left(P_{s 1}\right)$, which will be illustrated further by simulation results below.

In order to confirm the efficiency of the preceding analysis, extensive simulation experiments are performed to compare the simulation results of outage probability with the analytical results of the above lower and upper bounds. In the MonteCarlo simulations, all channels are subject to Rayleigh fading with unit mean and the variance of AWGNs at all nodes is set to unity. The power-allocation parameters at the secondary transmitter and the relay are off-line computed as per (19). Furthermore, for each channel realization, the optimal transmit powers at the secondary transmitter and the relay are determined by using $(9 \mathrm{c})$ and $(10 \mathrm{c})$, respectively.

On the one hand, the outage probability of the secondary receiver versus tolerable interference powers at primary receivers is illustrated in Figs. 2 and 3, where the average SNR at the secondary transmitter is fixed at $20 \mathrm{~dB}$ and the outage threshold is set to 0dB. In particular, Fig. 2 focuses on the scenarios with symmetric CCIs. That is, the CCI at

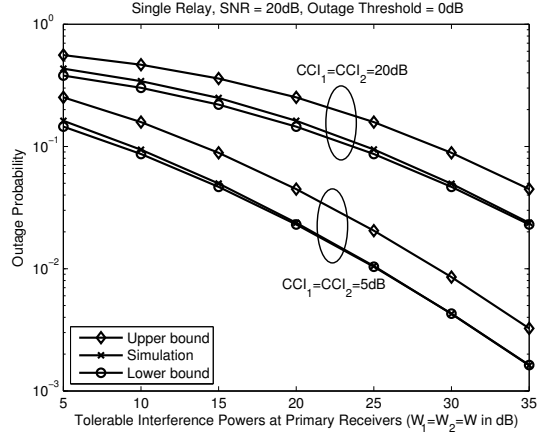

Fig. 2. Outage probability versus tolerable interference powers at primary receivers for the single-relay scenario with symmetric co-channel interferences.

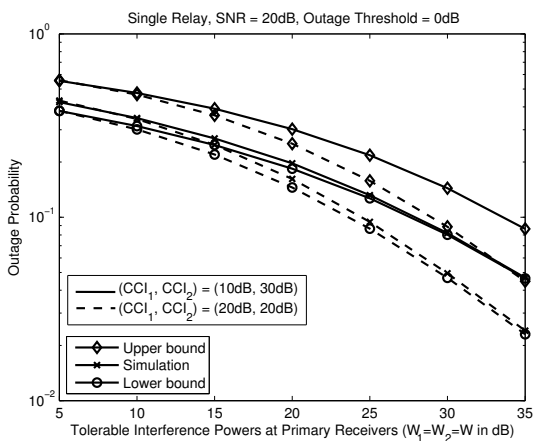

Fig. 3. Outage probability versus tolerable interference powers at primary receivers for the single-relay scenario with non-symmetric co-channel interferences, in comparison with the scenario with symmetric interferences.

the relay $\left(\mathrm{CCI}_{1}\right)$ is identical to that at the secondary receiver $\left(\mathrm{CCI}_{2}\right)$, i.e. $\mathrm{CCI}_{1}=\mathrm{CCI}_{2}$. It is observed from Fig. 2 that the simulation results of outage probability are very tight with the lower bound computed by (34). Moreover, outage probability decreases with increasing tolerable interference power $(W)$ at primary receivers, since larger value of $W$ allows higher transmit power at the secondary transmitter. It is also attested that outage probability deteriorates with higher $\mathrm{CCI}$ from concurrent primary transmission, which is in agreement with the observation in [19]. Figure 3 focuses on the scenario with non-symmetric CCIs, in contrast to the symmetric case with same sum of $\mathrm{CCI}_{1}$ and $\mathrm{CCI}_{2}$. It is observed that the simulation results are still close to the lower bound. Furthermore, it is seen that the non-symmetric scenario under-performs the symmetric scenario in terms of outage probability, since the end-to-end SIR is dominated by the SIR of the worse link between two consecutive hops, as suggested by (29).

On the other hand, the outage probability of the secondary receiver versus the average SNR at the secondary transmitter is illustrated in Figs. 4 and 5, where the tolerable interference powers at primary receivers are symmetric and are fixed at $20 \mathrm{~dB}$, and the outage threshold is set to $0 \mathrm{~dB}$. Specifically, Fig. 4 focuses on the scenarios with symmetric CCIs. It is observed that the simulation results are very tight with the lower bound computed by (34). Furthermore, the outage probability deteriorates with increasing CCIs introduced by primary transmitters, as expected. Figure 5 treats the scenario with non-symmetric CCIs, in contrast to the symmetric case with the same sum of $\mathrm{CCI}_{1}$ and $\mathrm{CCI}_{2}$. It is found that 


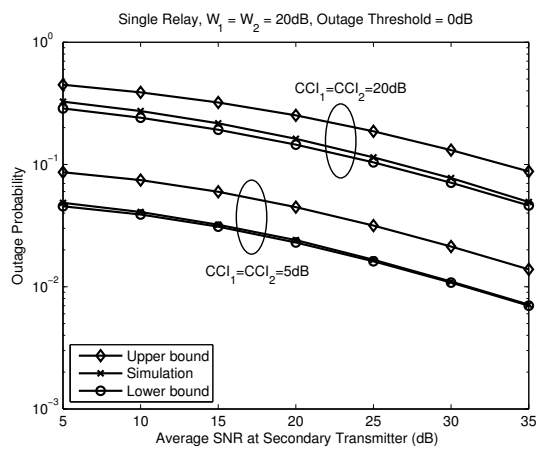

Fig. 4. Outage probability versus average SNR at the secondary transmitter for the single-relay scenario with symmetric co-channel interferences.

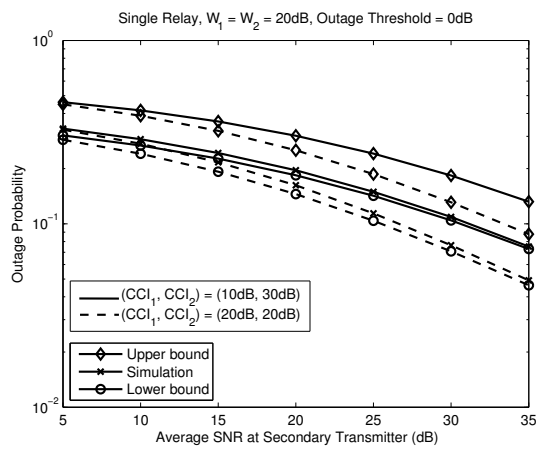

Fig. 5. Outage probability versus average SNR at the secondary transmitter for the single-relay scenario with non-symmetric co-channel interferences, in comparison with the scenario with symmetric interferences.

the simulation results are still close to the lower bound. Moreover, the non-symmetric scenario performs worse than the symmetric scenario, due to the same reason as described above.

Subsequently, Fig. 6 demonstrates the effect of CCIs from concurrent primary transmission on outage probability of the secondary receiver, where the average SNR at the secondary transmitter is fixed at $20 \mathrm{~dB}$ and the tolerable interference powers at primary receivers are set to $W_{1}=W_{2}=5 \mathrm{~dB}$ and $W_{1}=W_{2}=20 \mathrm{~dB}$. In this figure, the abscissa stands for the symmetric CCIs from primary transmitter (i.e. $P_{s 1}=P_{s 2}$ ) and it decreases from $35 \mathrm{~dB}$ to $5 \mathrm{~dB}$. It is observed that the simulation results of outage probability are tight with the lower bound computed by (34). Also, it is seen that outage probability improves with decreasing CCIs, as expected.

In the last but not the least, we compare Fig. 2, Fig. 4, and Fig. 6. It is observed that the curves in Fig. 2 demonstrate larger coding gain than the curves in Fig. 4 and Fig. 6. This means that the tolerable interference powers at primary receivers have more significant effect on outage probability than the average SNR at the secondary transmitter and the CCIs from primary transmitters. This is because the tolerable interference powers determine not only the transmit powers of the secondary transmitter and the relay, but also the gain of optimal power allocation. Furthermore, this observation is consistent with the preceding analysis on coding gain.

In summary, when there is only a single relay in the system under study, we conclude that, 1) it is the tolerable interference powers at primary receivers that dominate system

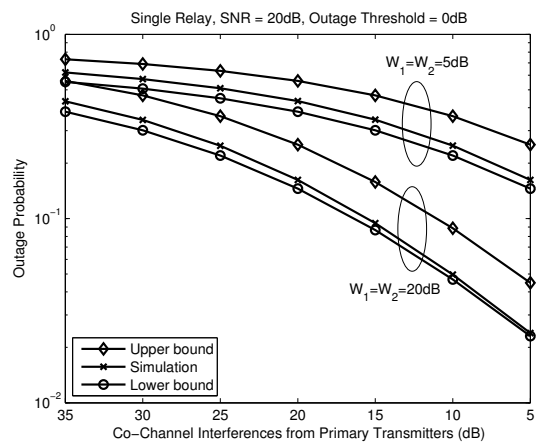

Fig. 6. Outage probability versus co-channel interferences for the single-relay scenario with symmetric tolerable interference powers at primary receivers.

performance, rather than the CCIs from concurrent primary transmission or the average SNR at the secondary transmitter; 2) the scenario with symmetric CCIs at the relay and the secondary receiver outperforms that with non-symmetric CCIs in terms of outage probability; 3) outage probability of the system is highly predictable from the lower bound that is analytically provided in (34) together with (23) and (26).

\section{B. Multi-Relay Scenario}

When there are $N>1$ relays available, opportunistic relay selection described in Section III-B can be exploited to achieve higher spatial diversity gain so as to improve the outage performance of secondary link without extra transmit power. Specifically, the relay $R_{i}, i=1, \cdots, N$, that has the maximum end-to-end SIR will be chosen to cooperate between the secondary transmitter and its receiver, while the other $N-1$ relays keep silent. Mathematically, the maximum endto-end SIR is given by

$$
\gamma_{\max }=\max _{i=1, \cdots, N} \gamma_{i}
$$

Substituting (29) into (42) yields the lower and upper bounds on $\gamma_{\max }$, given by

$$
\underbrace{\max _{i=1, \cdots, N} \gamma_{i-\text { low }}}_{\gamma_{\text {low }}} \leq \gamma_{\max } \leq \underbrace{\max _{i=1, \cdots, N} \gamma_{i-\text { up }}}_{\gamma_{\text {up }}} .
$$

Notice that, when there are multiple relaying nodes and opportunistic relay selection is adopted, the end-to-end SIRs are not independent of each other because of the common interference-power constraint at secondary transmitter and the common CCI at the secondary receiver (cf. $h_{0}$ and $q_{0}$ in Fig. 1-d)). Due to potential correlation effect, exact analysis of the lower and upper bounds given by (43) is mathematically intractable. ${ }^{2}$ In order to proceed, we relax the lower bound and approximate the upper bound shown in (43). In particular, the approximated upper bound will be shown to be very tight with simulation results. More specifically, the CDF of the lower

\footnotetext{
${ }^{2}$ As pointed out by one of the anonymous reviewers, the correlation between received SNRs in spectrum-sharing systems with relay selection was recently addressed in [12], [22], [23]. In such case, however, as stated by the authors of [12], [22], accurate analysis for the outage probability of the highest received SNR is mathematically intractable. [23] deals with the selection between direct link and relaying link with no relay selection.
} 


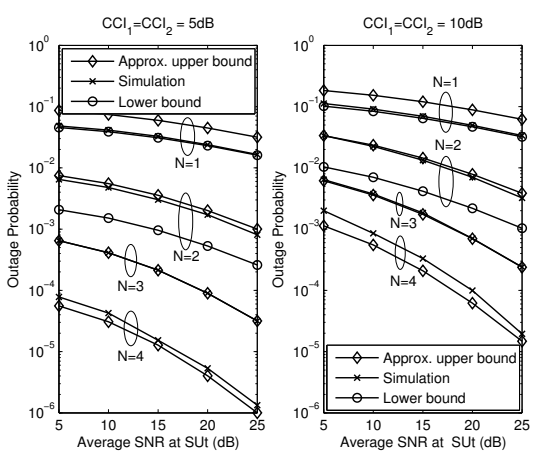

Fig. 7. The effect of opportunistic relay selection on outage probability of the secondary receiver.

bound $\gamma_{\text {low }}$ shown in (43) can be further bounded by

$$
\begin{aligned}
& F_{\gamma_{\text {low }}}(\gamma)<F_{\gamma_{\text {low }}^{\prime}}(\gamma) \\
& \quad=\left[F_{\gamma_{1 i}}(2 \gamma)+F_{\gamma_{2 i}}(2 \gamma)-F_{\gamma_{1 i}}(2 \gamma) F_{\gamma_{2 i}}(2 \gamma)\right]^{N},
\end{aligned}
$$

where $\gamma_{\text {low }}^{\prime} \triangleq \max _{i=1, \cdots, N} \gamma_{i-\text { low }}$ in which $\gamma_{i-\text { low }}, i=1, \cdots, N$ are assumed to be independent of each other, and (32) was exploited to derive the equality in (44). Similarly, the CDF of the upper bound $\gamma_{\text {up }}$ given in (43) can be further bounded by

$$
\begin{aligned}
& F_{\gamma_{\mathrm{up}}}(\gamma)<F_{\gamma_{\mathrm{up}}^{\prime}}(\gamma) \\
& \quad=\left[F_{\gamma_{1 i}}(\gamma)+F_{\gamma_{2 i}}(\gamma)-F_{\gamma_{1 i}}(\gamma) F_{\gamma_{2 i}}(\gamma)\right]^{N},
\end{aligned}
$$

where $\gamma_{\text {up }}^{\prime} \triangleq \max _{i=1, \cdots, N} \gamma_{i-\text { up }}$ in which $\gamma_{i-\text { up }}, i=1, \cdots, N$ are assumed to be independent of each other, and (33) was exploited to derive the equality in (45). As a result, recalling the fact that outage probability is a monotonically decreasing function of the end-to-end SIR, and substituting (44) and (45) into (43) yields the lower and upper bounds on the outage probability of the end-to-end SIR, given by

$$
\begin{aligned}
& {\left[F_{\gamma_{1 i}}\left(\gamma_{\mathrm{th}}\right)+F_{\gamma_{2 i}}\left(\gamma_{\mathrm{th}}\right)-F_{\gamma_{1 i}}\left(\gamma_{\mathrm{th}}\right) F_{\gamma_{2 i}}\left(\gamma_{\mathrm{th}}\right)\right]^{N}} \\
& \quad<\operatorname{Poultage}_{\text {outh }}\left(\gamma_{\mathrm{th}}\right) \lesssim \\
& \quad\left[F_{\gamma_{1 i}}\left(2 \gamma_{\mathrm{th}}\right)+F_{\gamma_{2 i}}\left(2 \gamma_{\mathrm{th}}\right)-F_{\gamma_{1 i}}\left(2 \gamma_{\mathrm{th}}\right) F_{\gamma_{2 i}}\left(2 \gamma_{\mathrm{th}}\right)\right]^{N},
\end{aligned}
$$

where the superscript "multi" of Pmulti $\left(\gamma_{\text {th }}\right)$ stands for the multi-relay case and the operator $\lesssim$ means "approximately less than".

Figure 7 illustrates the effect of opportunistic relay selection on outage probability versus the average SNR at the secondary transmitter. In the simulations, the tolerable interference powers at primary receivers are set to $20 \mathrm{~dB}$ (i.e. $W_{1}=W_{2}=20 \mathrm{~dB}$ ) and the CCIs from primary transmitters are symmetric with $\mathrm{CCI}_{1}=\mathrm{CCI}_{2}=5 \mathrm{~dB}$ on the left-hand side panel and $\mathrm{CCI}_{1}=\mathrm{CCI}_{2}=10 \mathrm{~dB}$ on the right-hand side panel. It is seen that, when the number of relays increases, outage probability decreases significantly due to higher spatial diversity gain. Essentially, by use of a similar approach as in [8], it is easy to show that the diversity order is $N$ when there are $N$ relays and opportunistic relay selection is adopted in the communication process between the secondary transmitter and its receiver. These different diversity orders correspond to the slopes of the curves associated with different number of relays in Fig. 7.
On the other hand, it is observed from Fig. 7 that, when there are $N=2$ or 3 relays, the simulation results of outage probability are very tight with the approximate upper bound offered by (46) in closed-form. However, when there are $N=4$ relays, the simulation results are slightly larger than the approximate upper bound. This implies that when the number of relays increases and opportunistic relay selection is adopted, the effect of the correlation between the end-to-end SIRs becomes more evident. In practice, an increased number of relays $(N)$ costs larger feedback overhead $\left(\log _{2} N\right)$ and, thus, it is widely accepted that up to four relays with 2-bit feedback overhead is feasible in real-world wireless systems with relay selection. Finally, it is remarkable that, when the number of relays increases from $N=1$ to $N>1$ and opportunistic relay selection is adopted, the observation that the outage probability is tight with the lower bound for the single-relay case and moves closer and closer to the upper bound when there are more and more relays available is not surprising. The reason behind this is as follows. In the single-relay case, the endto-end SIR approaches its upper bound shown in (29) due to the random fluctuations of channel fading, i.e. $\gamma_{1 i} \neq \gamma_{2 i}$. However, when there are more and more relays, the maximum end-to-end SIR approaches its lower bound shown in (43) since $\gamma_{1 i}$ and $\gamma_{2 i}$ are symmetric. A similar phenomenon can be observed, for example in [24, Fig. 6] and [25, Fig. 1].

In summary, since the transmit power at SUs in spectrumsharing systems is strictly limited and the CCIs from concurrent primary transmission are inevitable, opportunistic relay selection can be exploited to improve outage probability of secondary link significantly, and this probability is well predictable from the approximate upper bound analytically provided in (46).

\section{CONCLUSIONS}

In spectrum-sharing cooperative AF relaying systems, the effect of CCIs from concurrent primary transmission on the outage probability of secondary transmission was analytically investigated in this paper. In order to combat the CCIs, the transmit powers of a secondary transmitter and its relays were optimized with respect to both the average tolerable interference powers at primary receivers and the CCIs from primary transmitters. Subsequently, the lower and upper bounds on outage probability of the secondary receiver were explicitly derived. In particular, when there is only a single relay, the outage probability closely approaches the lower bound. When there are multiple relays, opportunistic relay selection can be exploited to further improve system performance and the outage probability is very tight with the upper bound. By analyzing these bounds on outage probability, this study reveals that it is the tolerable interference powers at PUs that dominate system performance of secondary transmission in spectrum-sharing context, even though the CCIs from PUs will degrade it.

\section{APPENDIX A}

PROOF OF LEMMA 1

Since the channel gains $\left|h_{0}\right|^{2},\left|q_{i}\right|^{2}$, and $\left|f_{i}\right|^{2}$ are assumed to be independent of each other, we can rewrite $V=\frac{\left|h_{0}\right|^{2}\left|q_{i}\right|^{2}}{\left|f_{i}\right|^{2}}$ 
as $V=X\left|q_{i}\right|^{2}$, where $X \triangleq \frac{\left|h_{0}\right|^{2}}{\left|f_{i}\right|^{2}}$. In the sequel, we first derive the PDF of $X$ and then the PDF of $V$.

Conditioning on $\left|f_{i}\right|^{2}$, the PDF of $X$ can be given by

$$
\begin{aligned}
f_{X}(x) & =\int_{0}^{\infty} f_{\left.X|| f_{i}\right|^{2}}(x \mid y) f_{\left|f_{i}\right|^{2}}(y) \mathrm{d} y \\
& =\int_{0}^{\infty} y f_{\left|h_{0}\right|^{2}}(x y) f_{\left|f_{i}\right|^{2}}(y) \mathrm{d} y \\
& =\frac{1}{\bar{\gamma}^{2}} \int_{0}^{\infty} y \exp \left[-\frac{1}{\bar{\gamma}}(x+1) y\right] \mathrm{d} y \\
& =(x+1)^{-2},
\end{aligned}
$$

where (12) was substituted into (47) to derive (48), and [16, Eq.(3.351.3)] was used to reach (49). Moreover, using a similar approach as in the above, the PDF of $V$ can be derived as

$$
\begin{aligned}
f_{V}(x) & =\int_{0}^{\infty} f_{\left.V|| q_{i}\right|^{2}}(x \mid y) f_{\left|q_{i}\right|^{2}}(y) \mathrm{d} y \\
& =\int_{0}^{\infty} \frac{1}{y} f_{X}\left(\frac{x}{y}\right) f_{\left|q_{i}\right|^{2}}(y) \mathrm{d} y \\
& =\frac{1}{\bar{\gamma}} \int_{0}^{\infty} \frac{y}{(x+y)^{2}} \exp \left(-\frac{y}{\bar{\gamma}}\right) \mathrm{d} y \\
& =\frac{1}{\bar{\gamma}} \Psi\left(2,1 ; \frac{x}{\bar{\gamma}}\right),
\end{aligned}
$$

where (12) was inserted again in (50) to derive (51), and [17, vol.1, Eq.(2.3.6.9)] was used to obtain (52). This leads to the intended result shown in (13).

\section{APPENDIX B}

PROOF OF THEOREM 1

According to (20), the CDF of the SIR $\gamma_{1 i}$ at the first hop can be expressed by definition as

$$
\begin{aligned}
& F_{\gamma_{1 i}}(\gamma) \\
& =\operatorname{Pr}\left\{\gamma_{1 i}=0\right\}+\operatorname{Pr}\left\{\gamma_{1 i}<\gamma \mid \gamma_{1 i}>0\right\}, \gamma>0 \\
& =\operatorname{Pr}\left\{\gamma_{1 i}=0\right\}+\frac{\operatorname{Pr}\left\{0<\gamma_{1 i}<\gamma\right\}}{\operatorname{Pr}\left\{\gamma_{1 i}>0\right\}} \\
& =\operatorname{Pr}\left\{V^{\prime} \leq \frac{P_{s 1}}{\lambda_{1}}\right\} \\
& \quad+\frac{\operatorname{Pr}\left\{V^{\prime}<\frac{P_{s 1}}{\lambda_{1}}(\gamma+1)\right\}-\operatorname{Pr}\left\{V^{\prime} \leq \frac{P_{s 1}}{\lambda_{1}}\right\}}{1-\operatorname{Pr}\left\{V^{\prime} \leq \frac{P_{s 1}}{\lambda_{1}}\right\}} \\
& =F_{V^{\prime}}\left(\frac{P_{s 1}}{\lambda_{1}}\right)+\frac{F_{V^{\prime}}\left(\frac{P_{s 1}}{\lambda_{1}}(\gamma+1)\right)-F_{V^{\prime}}\left(\frac{P_{s 1}}{\lambda_{1}}\right)}{1-F_{V^{\prime}}\left(\frac{P_{s 1}}{\lambda_{1}}\right)}
\end{aligned}
$$

where the second term $\operatorname{Pr}\left\{\gamma_{1 i}<\gamma \mid \gamma_{1 i}>0\right\}$ in the right-hand side of (53) denotes the conditional probability that $\gamma_{1 i}<\gamma$ occurs given that $\gamma_{1 i}>0$ has occurred, and (20) was exploited to derive (55). Then, differentiating (56) with respect to $\gamma$ yields the PDF of $\gamma_{1 i}$, given by

$$
f_{\gamma_{1 i}}(\gamma)=\frac{\frac{P_{s 1}}{\lambda_{1}} f_{V^{\prime}}\left(\frac{P_{s 1}}{\lambda_{1}}(\gamma+1)\right)}{1-F_{V^{\prime}}\left(\frac{P_{s 1}}{\lambda_{1}}\right)} .
$$

By virtue of (21), the term $F_{V^{\prime}}\left(\frac{P_{s 1}}{\lambda_{1}}\right)$ in the denominator of (57) can be computed as

$$
\begin{aligned}
& F_{V^{\prime}}\left(\frac{P_{s 1}}{\lambda_{1}}\right)=\int_{0}^{\frac{P_{s 1}}{\lambda_{1}}} \frac{1}{\bar{\gamma} x^{2}} \Psi\left(2,1 ; \frac{1}{\bar{\gamma} x}\right) \mathrm{d} x \\
& =\int_{0}^{\frac{\bar{\gamma} P_{s 1}}{\lambda_{1}}} \frac{1}{x^{2}} \Psi\left(2,1 ; \frac{1}{x}\right) \mathrm{d} x \\
& =\int_{\frac{\lambda_{1}}{\gamma_{P} P_{s 1}}}^{\infty} \Psi(2,1 ; x) \mathrm{d} x \\
& =\int_{0}^{\infty} \Psi(2,1 ; x) \mathrm{d} x \\
& -\int_{0}^{\frac{\lambda_{1}}{\bar{\gamma} P_{s 1}}} \Psi(2,1 ; x) \mathrm{d} x \\
& =1-\frac{\lambda_{1}}{\bar{\gamma} P_{s 1}} G_{2,3}^{2,2}\left[\begin{array}{c|c}
\lambda_{1} & 0,-1 \\
\bar{\gamma} P_{s 1} & 0,0,-1
\end{array}\right]
\end{aligned}
$$

where [16, Eq.(7.612.2)] was used to derive the first integral term in (61), and the second integral term in (61) was derived by using a similar approach as in (15)-(17). Consequently, substituting (62) into (57) yields

$$
f_{\gamma_{1 i}}(\gamma)=\frac{\bar{\gamma}}{G_{1}}\left(\frac{P_{s 1}}{\lambda_{1}}\right)^{2} f_{V^{\prime}}\left(\frac{P_{s 1}}{\lambda_{1}}(\gamma+1)\right)
$$

where $G_{1}$ denotes the Meijer's $G$-function given in (62). Finally, substituting (21) into (63) and performing some algebraic manipulations yields the intended PDF expression shown in (22).

On the other hand, based on the resulting PDF above, the CDF of $\gamma_{1 i}$ can be explicitly derived by definition as

$$
F_{\gamma_{1 i}}(\gamma)=\int_{0}^{\gamma} \frac{1}{(x+1)^{2} G_{1}} \Psi\left(2,1 ; \frac{c_{1}}{x+1}\right) \mathrm{d} x .
$$

By introducing the change of variable $y=\frac{1}{x+1}$, (64) can be rewritten as

$$
\begin{aligned}
F_{\gamma_{1 i}}(\gamma)= & \frac{1}{G_{1}} \int_{\frac{1}{\gamma+1}}^{1} \Psi\left(2,1 ; c_{1} y\right) \mathrm{d} y \\
= & \frac{1}{c_{1} G_{1}} \int_{\frac{c_{1}}{\gamma+1}}^{c_{1}} \Psi(2,1 ; x) \mathrm{d} x \\
= & \frac{1}{c_{1} G_{1}} \int_{\frac{c_{1}}{\gamma+1}}^{c_{1}} G_{1,2}^{2,1}\left[x \mid \begin{array}{c}
-1 \\
0,0
\end{array}\right] \mathrm{d} x \\
= & \frac{1}{G_{1}} G_{2,3}^{2,2}\left[c_{1} \mid \begin{array}{c}
0,-1 \\
0,0,-1
\end{array}\right] \\
& -\frac{1}{c_{1} G_{1}} G_{2,3}^{2,2}\left[\frac{c_{1}}{\gamma+1} \mid \begin{array}{c}
1,0 \\
\gamma+1,0
\end{array}\right],
\end{aligned}
$$

where (16) was employed to derive (67), and (14) and the scaling property of Meijer's $G$-function [17, vol.3, Eq.(8.2.2.15)] were used to derive (68). Finally, applying (24) to (68) leads to the desired CDF expression shown in (23), which completes the proof.

\section{REFERENCES}

[1] J. M. Peha, "Approaches to spectrum sharing," IEEE Commun. Mag., vol. 43, no. 2, pp. 10-12, Feb. 2005. 
[2] L. Musavian and S. Aïssa, "Cross-layer analysis of cognitive radio relay networks under quality of service constraints," in Proc. IEEE VTC'09spring, pp. 1-5, Barcelona, Spain, Apr. 2009.

[3] L. Musavian, S. Aïssa, and S. Lambotharan, "Effective capacity for interference and delay constrainted cognitive reatio relay channels," IEEE Trans. Wireless Commun., vol. 9, no. 5, pp. 1698-1707, May 2010.

[4] J. Lee, H. Wang, J. G. Andrews, and D. Hong, "Outage probability of cognitive relay networks with interference constraints," IEEE Trans. Wireless Commun., vol. 10, no. 2, pp. 390-395, Feb. 2011.

[5] V. Asghari and S. Aïssa, "End-to-end performance of cooperative relaying in spectrum-sharing systems with quality of service requirements," IEEE Trans. Veh. Technol., vol. 60, no. 6, pp. 2656-2668, June 2011.

[6] K. B. Fredj and S. Aïssa, "Performance of amplify-and-forward system with partial relay selection under spectrum-sharing constraints," IEEE Trans. Wireless Commun., vol. 11, no. 2, pp. 500-504, Feb. 2012.

[7] V. Asghari and S. Aïssa,"Performance of cooperative spectrum-sharing systems with amplify-and-forward relaying," IEEE Trans. Wireless Commun., vol. 11, no. 4, pp. 1295-1300, Apr. 2012

[8] M. Xia and S. Aïssa, "Cooperative AF relaying in spectrum-sharing systems: performance analysis under average interference power constraints and Nakagami-m fading," IEEE Trans. Commun., vol. 60, no. 6 , pp. 1523-1533, June 2012.

[9] S. Ikki and S. Aïssa, "Performance analysis of dual-hop relaying systems in the presence of co-channel interference," in Proc. IEEE Globecom'10, pp. 1-5, Miami, USA, Dec. 2010.

[10] L. Musavian and S. Aïssa, "Fundamental capacity limits of cognitive radio in fading environments with imperfect channel information," IEEE Trans. Commun., vol. 57, no. 11, pp. 3472-3480, Nov. 2009.

[11] L. Musavian and S. Aïssa, "Capacity and power allocation for spectrumsharing communications in fading channels," IEEE Trans. Wireless Commun., vol. 8, no. 1, pp. 148-156, Jan. 2009.

[12] J. Hong, B. Hong, T. Ban, and W. Choi, "On the cooperative diversity gain in underlay cognitive radio systems," IEEE Trans. Commun., vol. 60, no. 1, pp. 209-219, Jan. 2012.

[13] A. J. Goldsmith and P. P. Varajya, "Capacity of fading channels with channel side information," IEEE Trans. Inf. Theory, vol. 43, no. 6, pp. 1986-1992, Nov. 1997.

[14] A. Bletsas, A. Khisti, D. P. Reed, and A. Lippman, "A simple cooperative diversity method based on network path selection," IEEE J. Select. Area Commun., vol. 24, no. 3, pp. 659-672, Mar. 2006.

[15] A. Adinoyi, Y. Fan, H. Yanikomeroglu, H. V. Poor, and F. Al-Shaalan, "Performance of selection relaying and cooperative diversity," IEEE Trans. Wireless Commun., vol. 8, no. 12, pp. 5790-5795, Dec. 2009.

[16] I. S. Gradshteyn and I. M. Ryzhik, Table of Integrals, Series and Products, 7th Ed., Academic Press, 2007.

[17] A. P. Prudnikov, Y. A. Brychkov, and O. I. Marichev, Integrals and Series, Gordon and Breach Science Publishers, 1986.

[18] P. A. Anghel and M. Kaveh, "Exact symbol error probability of a cooperative network in a Rayleigh-fading environment," IEEE Trans. Wireless Commun., vol. 3, no. 5, pp. 1416-1421, May 2004.

[19] T. Q. Duong, V. N. Q. Bao, H. Tran, G. C. Alexandropoulos, and H.-J. Zepernick, "Effect of primary networks on the performance of spectrum sharing AF relaying," IET Electronics Lett., vol. 48, no. 1, pp. 25-27, Jan. 2012.

[20] Z. Wang and G. B. Giannakis, "A simple and general parameterization quantifying performance in fading channels," IEEE Trans. Commun. vol. 51, no. 8, pp. 1389-1398, Aug. 2003.

[21] L. C. Andrews, Special Functions of Mathematics for Engineers, 2nd Ed., McGraw-Hill, Inc., 1992.

[22] L. Luo, P. Zhang, G. Zhang, and J. Qin, "Outage performance for cognitive relay networks with underlay spectrum sharing," IEEE Commun. Letters, vol. 15, no. 7, pp. 710-712, July 2011.

[23] T. Q. Duong, V. N. Q. Bao, G. C. Alexandropoulos, and H. J. Zepernick, "Cooperative spectrum sharing networks with AF relay and selection diversity," IET Electronics Lett., vol. 47, no. 20, pp. 11491151, Sep. 2011.

[24] K.-S. Hwang, Y.-C. Ko, and M.-S. Alouini, "Performance analysis of incremental opportunistic relaying over identically and non-identically distributed cooperative paths," IEEE Trans. Wireless Commun., vol. 8, no. 4, pp. 1953-1961, Apr. 2009.

[25] Q. F. Zhou and F. C. M. Lau, "Performance bounds of opportunistic cooperative communications with CSI-assisted amplify-and-forward re- laying and MRC reception," IEEE Trans. Veh. Technol., vol. 59, no. 5, pp. 2159-2165, June 2010

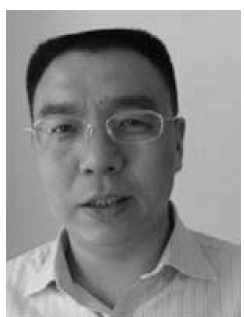

Minghua Xia (M'12) obtained his Ph.D. degree in Telecommunications and Information Systems from Sun Yat-sen University, Guangzhou, China, in 2007 From Mar. 2007 to July 2009, he was with the Electronics and Telecommunications Research Institute (ETRI) of South Korea, Beijing R\&D Center, Beijing, China, where he worked as a member of engineering staff and participated in the projects on the physical layer design of 3GPP LTE mobile communications. From Aug. 2009 to Feb. 2011, he was with The University of Hong Kong (HKU), Hong Kong, as a Postdoctoral Fellow. Currently, he is with King Abdullah University of Science and Technology (KAUST), Saudi Arabia. His research interests are in the area of network information theory and space-time signal processing, and in particular the design and performance analysis of multiuser multi-antenna systems, cooperative relaying systems, and cognitive radio networks.

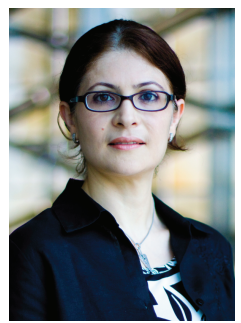

Sonia Aïssa (S'93-M'00-SM'03) received her Ph.D degree in Electrical and Computer Engineering from McGill University, Montreal, QC, Canada, in 1998. Since then, she has been with the National Institute of Scientific Research-Energy, Materials, and Telecommunications (INRS-EMT), University of Quebec, Montreal, QC, Canada, where she is a Full Professor.

From 1996 to 1997, she was a Researcher with the Department of Electronics and Communications of Kyoto University, Kyoto, Japan, and with the Wireless Systems Laboratories of NTT, Kanagawa, Japan. From 1998 to 2000 , she was a Research Associate at INRS-EMT, Montreal. From 2000 to 2002, while she was an Assistant Professor, she was a Principal Investigator in the major program of personal and mobile communications of the Canadian Institute for Telecommunications Research (CITR), leading research in radio resource management for code division multiple access systems. From 2004 to 2007, she was an Adjunct Professor with Concordia University, Montreal. In 2006, she was Visiting Invited Professor with the Graduate School of Informatics, Kyoto University, Kyoto, Japan. Her research interests lie in the area of wireless and mobile communications, and include radio resource management, cross-layer design and optimization, design and analysis of multiple antenna (MIMO) systems, cognitive and cooperative transmission techniques, and performance evaluation, with a focus on Cellular, Ad Hoc, and Cognitive Radio networks.

Dr. Aïssa was the Founding Chair of the Montreal Chapter IEEE Women in Engineering Society in 2004-2007, acted or is currently acting as Technical Program Leading Chair or Cochair for the Wireless Communications Symposium of the IEEE International Conference on Communications (ICC) in 2006, 2009, 2011 and 2012, as PHY/MAC Program Chair for the 2007 IEEE Wireless Communications and Networking Conference (WCNC), and as Technical Program Committee Cochair of the 2013 IEEE Vehicular Technology Conference - spring (VTC). She has served as a Guest Editor of the EURASIP Journal on Wireless Communications and Networking in 2006, and as Associate Editor of the IEEE WIRELESS COMMUNICATIONS MAGAZINE in 20062010. She is currently an Editor of the IEEE TRANSACTIONS ON WIRELESS COMMUNICATIONS, the IEEE TRANSACTIONS ON COMMUNICATIONS and the IEEE COMMUNICATIONS MAGAZINE, and Associate Editor of the Wiley Security and Communication Networks Journal. Awards and distinctions to her credit include the Quebec Government FQRNT Strategic Fellowship for Professors-Researchers in 2001-2006; the INRS-EMT Performance Award in 2004 and 2011 for outstanding achievements in research, teaching and service; the IEEE Communications Society Certificate of Appreciation in 2006-2012; and the Technical Community Service Award from the FQRNT Center for Advanced Systems and Technologies in Communications (SYTACom) in 2007. She is also co-recipient of Best Paper Awards from IEEE ISCC 2009, WPMC 2010, IEEE WCNC 2010, IEEE ICCIT 2011 and IEEE VTC 2011 (Kansai Section); and recipient of NSERC (Natural Sciences and Engineering Research Council of Canada) Discovery Accelerator Supplement Award. 